\title{
Geometria no conjunto das matrizes
}

\section{Geometry on matrices set}

Laerte Bemm

Universidade Estadual de Maringá (UEM), Departamento de Matemática (DMA), Programa de Mestrado Profissional em Matemática em Rede Nacional (PROFMAT), Maringá, PR, Brasil http://orcid.org/0000-0002-0326-7662, 1bemm20uem.br

Douglas Monteiro Caetano

Colégio Bertoni de Medianeira LTDA, Medianeira, PR, Brasil http://orcid.org/0000-0002-2104-0808, douglas555mcaetano@gmail.com

\section{Informações do Artigo}

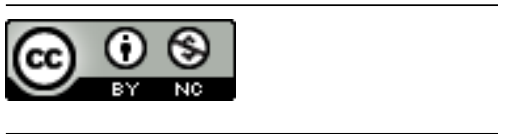

\section{Histórico do Artigo}

Submissão: 13 de março de 2019.

Aceite: 20 de maio de 2019.

\section{Palavras-chave}

Números Complexos

Matrizes

Produto Interno

Ângulo entre Matrizes

Módulo de Matrizes

\section{Resumo}

Este trabalho pretende estabelecer uma correspondência biunívoca entre o conjunto dos números complexos e um subconjunto $S$ de $M_{2 \times 2}(\mathbb{R})$ (matrizes $2 \times 2 \mathrm{com}$ entradas reais) e mostrar que esses conjuntos se comportam algebricamente da mesma forma. A partir disso foram definidos alguns elementos geométricos no conjunto das matrizes $M_{2 \times 2}(\mathbb{R})$. Mais precisamente, definimos o módulo de uma matriz e o ângulo entre duas matrizes. Em seguida, mostramos que a correspondência citada preserva ângulos e módulos.

\section{Keywords}

Complex Numbers

Matrices

Inner Product

Angle between Matrices

Module of Matrices

\begin{abstract}
This work intends to establish an one-to-one correspondence between the set of complex numbers and a subset $S$ of $M_{2 \times 2}(\mathbb{R})$ (matrices $2 \times 2$ with real entries) and show that these sets behave algebraically in the same way. From this, some geometric elements in the set of matrices $M_{2 \times 2}(\mathbb{R})$ were defined. More precisely, we define the module of a matrix and the angle between two matrices. Next, we show that the quoted correspondence preserves angles and modules.
\end{abstract}

\section{Introdução}

Os conceitos de segmento e ângulo são essenciais para o desenvolvimento da Geometria Euclidiana como conhecemos. Além disso, é a noção de medida destes entes geométricos que nos leva a definir congruência, semelhança e proximidade, bem como nos permite calcular áreas e volumes de figuras geométricas.

A partir da introdução da Geometria Analítica por René Descartes no século XVII, calcular a medida de um segmento ficou bastante fácil, bastando saber as coordenados das extremidades de 
tal segmento e aplicar o Teorema de Pitágoras. De fato, dado um ponto $P$ de coordenadas $(x, y)$ num sistema cartesiano ortogonal de coordenadas de $\mathbb{R}^{2}$, pelo Teorema de Pitágoras, a distância $d$ de $P$ até a origem $O$ do sistema é dada por $d=\sqrt{x^{2}+y^{2}}$. Também podemos interpretar este resultado dizendo que o comprimento do segmento $O P$ é $\sqrt{x^{2}+y^{2}}$.

Ainda com o domínio da Geometria Analítica, René Descartes estudou as equações algébricas e em uma passagem do Discurso do Método, ele escreveu: "Nem sempre as raízes verdadeiras (positivas) ou falsas (negativas) de uma equação são reais. Às vezes elas são imaginárias" (GARBI, 1997, p. 75). Devido a isto, até hoje $\sqrt{-1}$ é chamado de número imaginário e este se consagrou juntamente com a expressão "número complexo", devido a Carl F. Gauss. Já no século XVIII, Leonhard Euler contribuiu significativamente com o estudo dos números complexos e propôs trocar a notação de $\sqrt{-1}$ por $i$.

Podemos afirmar que a representação geométrica de um número complexo foi um dos grandes passos no estudo destes números. Em 1797, o dinamarquês Caspar Wessel foi o primeiro a fazer tal representação, estabelecendo uma correspondência biunívoca entre o conjunto dos números complexos e o conjunto dos pontos do plano $\mathbb{R}^{2}$. A partir disso, podemos definir alguns elementos geométricos para os números complexos, como o comprimento (ou módulo), argumento de um número complexo, e o ângulo entre números complexos.

O nosso objetivo é estabelecer uma correspondência biunívoca entre o conjunto dos números complexos e um subconjunto $S$ das matrizes $2 \times 2$ com entradas reais e mostrar que estes conjuntos tem o mesmo comportamento algébrico. A partir disso, definimos alguns elementos geométricos no conjunto das matrizes $M_{2 \times 2}(\mathbb{R})$. Mais precisamente, definimos o comprimento (norma ou módulo) de uma matriz e o ângulo entre duas matrizes. Nós finalizamos o trabalho mostrando que a correspondência citada preserva ângulos e comprimentos.

Para atingirmos nossos objetivos, dividimos nosso trabalho em 5 seções. Nas duas primeiras, nós fazemos uma revisão rápida sobre o conjuntos dos números complexos e o conjunto das matrizes. Na Seção 3 nós estabelecemos a correspondência bijetora citada no parágrafo anterior. Nas seção 4 nós definimos comprimento (ou norma) de uma matriz e ângulo entre matrizes e mostramos que a correspondência definida na Seção 3 também preserva estes entes. Na última seção, nós fazemos algumas considerações que achamos pertinentes. 


\section{O conjunto dos números complexos}

Nesta seção abordaremos o conjunto dos números complexos, os quais apresentamos como o conjunto $\mathbb{R} \times \mathbb{R}$, munido com uma estrutura algébrica de adição e multiplicação. Também apresentamos as noções de norma, argumento e ângulo entre números complexos. Para maiores detalhes e demonstrações que não apresentamos, veja, por exemplo, Fernandes e Bernardes (2008), Caetano (2018), Silva (2017), entre outros. Uma breve história sobre o surgimento dos números complexos pode ser encontrada em Gilberto (2009).

Seja $\mathbb{C}=\mathbb{R} \times \mathbb{R}=\{(x, y) ; x \in \mathbb{R}$ e $y \in \mathbb{R}\}$. Definimos:

1 - Igualdade: dois elementos $(a, b)$ e $(c, d)$ de $\mathbb{C}$ são iguais se, e somente se, $a=c$ e $b=d$.

2 - Adição: é uma operação em $\mathbb{C}$ que associa a cada par de elementos $(a, b),(c, d) \in \mathbb{C}$ o elemento $(a, b)+(c, d)=(a+c, b+d) \in \mathbb{C}$ chamado soma de $(a, b)$ e $(c, d)$.

3 - Multiplicação: é uma operação em $\mathbb{C}$ que associa a cada par $(a, b),(c, d) \in \mathbb{C}$ um único elemento $(a, b) \cdot(c, d)=(a c-b d, a d+b c)$ chamado produto. Algumas vezes $(a, b) \cdot(c, d)$ é escrito por $(a, b)(c, d)$.

4 - Multiplicação por um escalar: é a operação (externa) que associa um número $\lambda \in \mathbb{R}$ e um número complexo $(a, b) \in \mathbb{C}$ a um único elemento $\lambda(a, b)=(\lambda a, \lambda b) \in \mathbb{C}$, chamado produto por escalar.

O conjunto $\mathbb{C}$ com estas operações é chamado de Conjunto dos Números Complexos.

Para facilitar a escrita, representaremos os números complexos $(0,0)$ e $(1,0)$ por 0 e 1 , respectivamente. Ainda, para cada número $z=(a, b) \in \mathbb{C}, z \neq 0$, definimos

$$
z^{\prime}=\left(\frac{a}{a^{2}+b^{2}}, \frac{-b}{a^{2}+b^{2}}\right) .
$$

É fácil verificar que $z z^{\prime}=z^{\prime} z=(1,0)=1$. Por este motivo, $z^{\prime}$ é denotado por $z^{-1}$ e chamado inverso de $z$. Também, para cada $z=(a, b) \in \mathbb{C}$ definimos $-z=(-a,-b)$. Claramente, $z+(-z)=0$.

Com as operações de adição e multiplicação em $\mathbb{C}$, definimos as operações de subtração e divisão. Dados $z, w \in \mathbb{C}$ definimos $z-w=z+(-w)$ e $\frac{z}{w}=z w^{-1}$, sempre que $w \neq 0$. Também podemos identificar qualquer número real $x$ com o número complexo $(x, 0)$.

Observe que $(0,1)^{2}=(0,1) \cdot(0,1)=(0 \cdot 0-1 \cdot 1,0 \cdot 1+1 \cdot 0)=(-1,0)=-(1,0)=-1$, ou seja, há um número complexo que elevado ao quadrado resulta em -1 . O número complexo $(0,1)$ 
é denotado por $i$ e é chamado de número imaginário. Assim, $i^{2}=-1$. Além disso, para qualquer $z=(a, b) \in \mathbb{C}, z=(a, 0)+(0, b)=a(1,0)+b(0,1)=a+b i$. Tal expressão de $z$ é chamada forma algébrica de $z$. O número $a$ é dito a parte real de $z$ e denotamos por $\operatorname{Re}(z)$, enquanto que $b$ é a parte imaginária de $z$ e denotada por $\operatorname{Im}(z)$. Note que as operações de adição e multiplicação dos números complexos na forma algébrica são dadas por

$$
\begin{aligned}
z+w & =(a+b i)+(c+d i)=(a+c)+(b+d) i \\
& \mathrm{e} \\
z w & =(a+b i)(c+d i)=(a c-b d)+(a d+c b) i .
\end{aligned}
$$

Todo $z=(a, b) \in \mathbb{C}$ pode ser representado geometricamente no plano cartesiano, o qual chamamos, simplesmente, de plano complexo. Este plano possui dois eixos orientados e ortogonais, em que o eixo horizontal é chamado de eixo real e o eixo vertical de eixo imaginário. Chamamos de origem e denotamos por $\mathbf{O}$ o ponto de interseção destes eixos. A representação do número complexo $a+b i$ é dado por uma flecha de origem em $\mathbf{O}$ e ponto final no ponto $A$ de coordenadas $(a, b)$.

Dado um número complexo $z=a+b i$, definimos o conjugado de $z$ e indicamos por $\bar{z} 0$ número complexo que se obtém conservando a parte real e trocando o sinal da parte imaginária de $z$, ou seja, $\bar{z}=a-b i$. Se representarmos $z$ no plano complexo, $\bar{z}$ é representado através da reflexão de $z$ em relação ao eixo real. Por exemplo, para $z=-3+4 i$, temos $\bar{z}=-3-4 i$ e suas representações geométricas são dadas na figura a seguir.

Figura 1: Representação gráfica do número complexo $z=-3+4 i$ e seu conjugado.

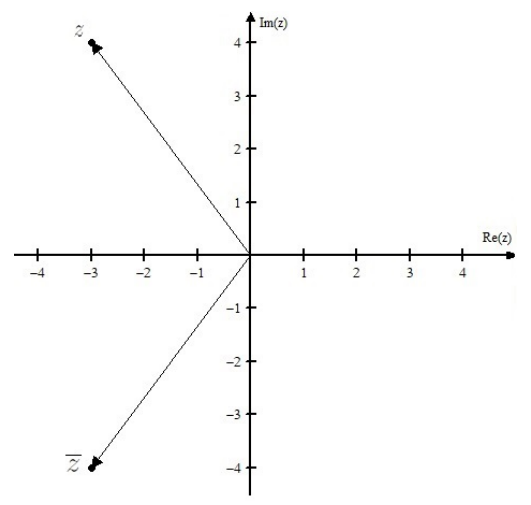

Fonte: Elaboração dos autores.

É fácil verificar que para quaisquer $z, w \in \mathbb{C}, \overline{\bar{z}}=z ; \overline{z \pm w}=\bar{z} \pm \bar{w}$ e $\overline{z w}=\bar{z} \bar{w}$. 


\subsection{Módulo de números complexos}

Dado um número complexo $z=a+b i$, os pontos $O, A$ e $B$ de coordenadas $(0,0),(a, 0)$ e $(a, b)$, respectivamente, determinam um triângulo retângulo em $A$. Então, pelo Teorema de Pitágoras, o comprimento do segmento $O B$ é dado por $\sqrt{a^{2}+b^{2}}$. Este número é denotado por $|z|$ e chamado comprimento, módulo ou norma do número complexo $z=a+b i$.

Figura 2: Representação gráfica do módulo de $z$.

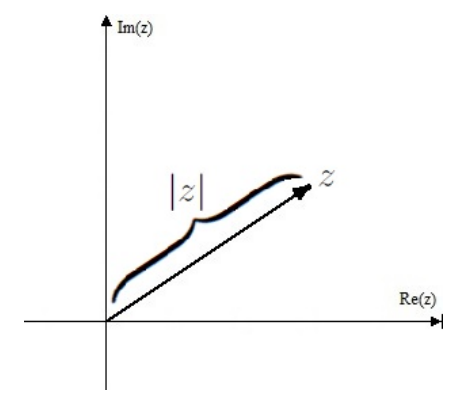

Fonte: Elaboração dos autores.

Aplicando-se a definição de norma, podemos verificar que para quaisquer $z, w \in \mathbb{C}$, temos $|z|^{2}=z \bar{z} ;|\bar{z}|=|z| ;|z w|=|z||w|$ e $|z / w|=|z| /|w|$, se $w \neq 0$. Além disso, $|z+w| \leq|z|+|w|$. Esta propriedade é chamada Desigualdade Triangular.

\section{2 Ângulo entre números complexos}

Seja $z=a+b i \in \mathbb{C}, \operatorname{com} z \neq 0$. Definimos 0 argumento de $z$ e denotamos por $\arg (z) \circ$ ângulo $\theta$ formado pelo eixo real positivo com a flecha correspondente a $z$ no sentido anti-horário.

Figura 3: Representação do ângulo de $z$.

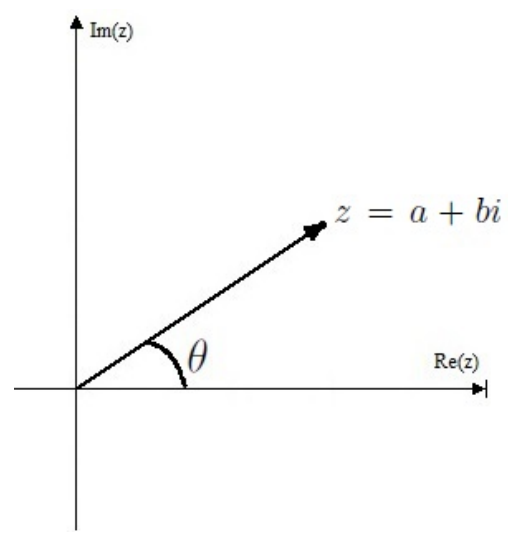

Fonte: Elaboração dos autores. 
Se $z=a+b i$, então da Trigonometria do Triângulo Retângulo, obtemos $a=|z| \cos \theta$ e $b=|z| \operatorname{sen} \theta$ e daí temos que

$$
z=|z|(\cos \theta+i \operatorname{sen} \theta), \operatorname{com} \theta \in \mathbb{R}
$$

Como as funções cosseno e seno são de período igual a $2 \pi$, temos que a Equação (1) continua válida para todo $\theta+2 k \pi$, em que $k \in \mathbb{Z}$. Com isso, podemos afirmar que existem infinitos argumentos de $z$ que satisfazem tal equação. Porém, no intervalo real $[\theta, \theta+2 \pi)$, há unicidade. Por isso, consideraremos $\arg (z)$ no intervalo $[0,2 \pi)$. Logo, $z=|z|(\cos \arg (z)+i \operatorname{sen} \arg (z))$, chamada a forma polar de $z$.

Considere $z_{1}=a+b i, z_{2}=c+d i$ dois números complexos não nulos e sejam $A_{1}$ de coordenadas $(a, b)$ e $A_{2}$ de coordenadas $(c, d)$. Seja $\theta$ o ângulo entre $A_{1} O A_{2}$ cuja medida pertence a $[0, \pi]$. Definimos $\theta$ como sendo o ângulo entre $z_{1}$ e $z_{2}$ e o denotamos por $\theta_{z_{1}, z_{2}}$. Observe que se $\arg \left(z_{2}\right)-\arg \left(z_{1}\right) \in[0, \pi]$, então o ângulo entre $z_{1}$ e $z_{2}$ é $\arg \left(z_{2}\right)-\arg \left(z_{1}\right)$. Porém, se $\arg \left(z_{2}\right)-\arg \left(z_{1}\right)>\pi$, então o ângulo entre $z_{1}$ e $z_{2}$ é $2 \pi-\left(\arg \left(z_{2}\right)-\arg \left(z_{1}\right)\right)$.

Usando propriedades de trigonometria, podemos provar que $\cos \left(\theta_{z_{1}, z_{2}}\right)=\frac{a c+b d}{\sqrt{a^{2}+b^{2}} \sqrt{c^{2}+d^{2}}}$.

Figura 4: Ângulo entre dois números complexos.

(a) $\theta=\arg \left(z_{2}\right)-\arg \left(z_{1}\right)$

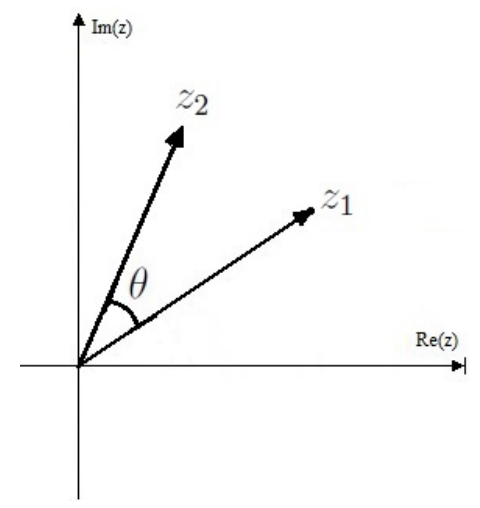

(b) $\theta=2 \pi-\left(\arg \left(z_{2}\right)-\arg \left(z_{1}\right)\right)$

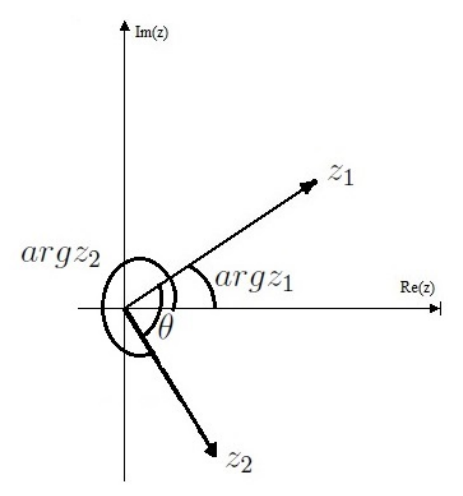

Fonte: Elaboração dos autores.

\section{Matrizes}

Chamamos de matriz uma tabela de elementos dispostos em linhas e colunas. Os elementos que compõem cada matriz são chamados de entradas. 
A representação de uma matriz com $m$ linhas e $n$ colunas é dada por

$$
A=\left(\begin{array}{cccc}
a_{11} & a_{12} & \cdots & a_{1 n} \\
\vdots & \vdots & & \vdots \\
a_{m 1} & a_{m 2} & \cdots & a_{m n}
\end{array}\right)
$$

em que cada $a_{i j} \in \mathbb{R}$, com $1 \leq i \leq m$ e $1 \leq j \leq n$. Observe que os índices $i$ e $j$ indicam a posição do elemento $a_{i j}$ na matriz. O conjunto de todas as matrizes de $m$ linhas e $n$ colunas com entradas reais é denotado por $\mathbb{M}_{m \times n}(\mathbb{R})$. Também usamos a notação $A=\left[a_{i j}\right]_{m \times n}$ ou $A_{m \times n}=\left[a_{i j}\right]_{m \times n}$ para indicar uma matriz de $\mathbb{M}_{m \times n}(\mathbb{R})$.

Definição 3.1. Duas matrizes $A=\left[a_{i j}\right]_{m \times n}$ e $B=\left[b_{i j}\right]_{r \times s}$ são iguais, escrevemos $A=B$, se elas têm o mesmo número de linhas $(m=r)$ e colunas $(n=s)$ e todos os seus elementos correspondentes são iguais $\left(a_{i j}=b_{i j}\right)$.

Definição 3.2. Dada uma matriz $A=\left[a_{i j}\right]_{m \times n}$, a matriz oposta de $A$ é a matriz $-A=\left[-a_{i j}\right]_{m \times n}$.

Entre a infinidade de matrizes que podemos escrever, existem aquelas que são frequentemente usadas. Vamos listar algumas.

Matriz Quadrada: é toda matriz cujo número $m$ de linhas é igual ao número $n$ de colunas $(m=n)$. Neste caso dizemos que a matriz é de ordem $m$ (ou $n$ ).

Matriz Nula: é qualquer matriz que tem todas as entradas nulas. Usaremos $\mathbf{0}$ para denotar uma matriz nula, independentemente da ordem.

Matriz Identidade: é a matriz quadrada $I_{n}=\left[a_{i j}\right]_{n \times n}$ em que $a_{i i}=1$, para todo $i=1, \ldots, n \mathrm{e}$ $a_{i j}=0$, para $i \neq j$.

Estes e outros tipos de matrizes podem ser encontrados na Seção 1.2 de BOLDRINI (1986).

\subsection{Operações com matrizes}

Para trabalhamos com matrizes, é necessário definirmos operações. Nesta seção definimos algumas operações e apresentamos certas propriedades que serão utilizadas em seções posteriores. As definições e resultados que apresentamos nessa seção são bem conhecidos. As demonstrações podem ser encontradas no Capítulo 1 de Callioli (1978). 
Definição 3.3. A adição de duas matrizes de mesma ordem, $A=\left[a_{i j}\right]_{m \times n}$ e $B=\left[b_{i j}\right]_{m \times n}$, é uma matriz $m \times n$, que denotaremos $A+B$, cujos elementos são somas dos elementos correspondentes de $A$ e $B$, isto é, $A+B=\left[a_{i j}+b_{i j}\right]_{m \times n}$.

Dadas quaisquer matrizes $A, B$ e $C$ de mesma ordem $m \times n$, temos: $A+B=B+A$; $A+(B+C)=(A+B)+C ; A+\mathbf{0}=A$ e $A+(-A)=\mathbf{0}$.

Definição 3.4. Sejam $A=\left[a_{i j}\right]_{m \times n} \in \mathbb{M}_{m \times n}(\mathbb{R})$ e $k$ um número real. Definimos a multiplicação de $A$ por $k$ como a matriz denotada por $k A$ e dada por $k A=\left[k a_{i j}\right]_{m \times n}$.

Proposição 3.5. Dadas matrizes $A$ e $B$ de mesma ordem $m \times n$ e $k_{1}, k_{2}, k \in \mathbb{R}$, temos:

i) $k(A+B)=k A+k B$;

ii) $\left(k_{1}+k_{2}\right) A=k_{1} A+k_{2} A$;

iii) $0 A=\mathbf{0}$;

iv) $k_{1}\left(k_{2} A\right)=\left(k_{1} k_{2}\right) A$.

Definição 3.6. Sejam $A=\left[a_{i j}\right]_{m \times n}$ e $B=\left[b_{r s}\right]_{n \times p}$ matrizes com entradas reais. Definimos a multiplicação da matriz $A$ pela matriz $B$ como sendo a matriz $A B=\left[c_{u v}\right]_{m \times p}$ em que

$$
c_{u v}=\sum_{k=1}^{n} a_{u k} b_{k v}=a_{u 1} b_{1 v}+\cdots+a_{u n} b_{n v} .
$$

A matriz $A B$ é chamada produto de $A$ por $B$.

Observação 3.7. Só podemos efetuar o produto de duas matrizes $A_{m \times n}$ e $B_{l \times p}$ se o número de colunas da primeira for igual ao número de linhas da segunda, isto é, $n=l$. Além disso, a matriz resultante será de ordem $m \times p$.

Proposição 3.8. Quando forem possíveis as operações, as seguintes propriedades são válidas para quaisquer matrizes $A, B, C$ :

i) $(A B) C=A(B C)$;

ii) $A(B+C)=A B+A C$;

iii) $(A+B) C=A C+B C$;

iv) $\mathbf{0} A=\mathbf{0}=A \mathbf{0}$; 
v) Se $A$ é um matriz quadrada de ordem $n, A I_{n}=A=I_{n} A$.

Dada uma matriz $A=\left[a_{i j}\right]_{m \times n}$, podemos obter outra matriz $A^{t}=\left[b_{i j}\right]_{n \times m}$, cuja $i$-ésima linha é a $i$-ésima coluna de $A$, isto é, $b_{i j}=a_{j i}$. A matriz $A^{t}$ é denominada transposta de $A$.

Exemplo 3.9. Se $A=\left(\begin{array}{rr}2 & 0 \\ 3 & 1 \\ -5 & -7\end{array}\right)$, então $A^{t}=\left(\begin{array}{rrr}2 & 3 & -5 \\ 0 & 1 & -7\end{array}\right)$.

Definição 3.10. Definimos o traço como sendo a função $t r: \mathbb{M}_{m}(\mathbb{R}) \longrightarrow \mathbb{R}$ que associa a cada matriz $A=\left[a_{i j}\right]_{m \times m} \in \mathbb{M}_{m}(\mathbb{R})$ o número real $a_{11}+\cdots+a_{m m}$, ou seja,

$$
\operatorname{tr}(A)=a_{11}+\cdots+a_{m m}
$$

Proposição 3.11. Considere as matrizes quadradas $A=\left[a_{i j}\right]_{m \times m}$ e $B=\left[b_{i j}\right]_{m \times m}$, e a matriz identidade $I_{m}$. As seguintes propriedades são válidas:

(i) $\operatorname{tr}(A)=\operatorname{tr}\left(A^{t}\right)$;

(ii) $\operatorname{tr}\left(I_{m}\right)=m$;

(iii) $\operatorname{tr}(k A)=k \operatorname{tr}(A)$;

(iv) $\operatorname{tr}(A B)=\operatorname{tr}(B A)$.

\section{Uma relação entre números complexos e matrizes}

Nesta seção apresentamos uma maneira de identificar o conjunto dos números complexos $\mathbb{C}$ com um subconjunto $S$ de $\mathbb{M}_{2 \times 2}(\mathbb{R})$ via uma função bijetora. Mostraremos também que $\mathbb{C}$ e $S$ se comportam algebricamente de maneira idêntica. Essa identificação é bem conhecida entre os matemáticos (veja, por exemplo, Soares (2009)). Abordagens muito mais completas e detalhadas podem ser encontradas em Caetano (2018) e Silva (2017).

Considere a matriz $A=\left(\begin{array}{cc}0 & 1 \\ -1 & 0\end{array}\right) \in \mathbb{M}_{2 \times 2}(\mathbb{R})$. É fácil ver $A^{2}=-I_{2}$. Pela Proposição 3.8(v), $I_{2}$ desempenha em $\mathbb{M}_{2 \times 2}(\mathbb{R})$ o mesmo papel que 1 desempenha em $\mathbb{C}$. Isto significa que em $\mathbb{M}_{2 \times 2}(\mathbb{R})$ também há um elemento cujo quadrado é igual a "menos um". Por isso, seja $S$ o conjunto das matrizes da forma $a\left(\begin{array}{ll}1 & 0 \\ 0 & 1\end{array}\right)+b\left(\begin{array}{cc}0 & 1 \\ -1 & 0\end{array}\right), \operatorname{com} a, b \in \mathbb{R}$, i. é, $S=\left\{\left(\begin{array}{cc}a & b \\ -b & a\end{array}\right) ; a, b \in \mathbb{R}\right\}$. 
Note que as matrizes identidade $\left(\begin{array}{ll}1 & 0 \\ 0 & 1\end{array}\right)$ e nula $\left(\begin{array}{ll}0 & 0 \\ 0 & 0\end{array}\right)$ de ordem 2 pertencem a $S$. Além disso, se $A=\left(\begin{array}{cc}a & b \\ -b & a\end{array}\right)$ e $B=\left(\begin{array}{cc}c & d \\ -d & c\end{array}\right)$ são elementos de $S$ e $k \in \mathbb{R}$, então

$$
\begin{gathered}
A+B=\left(\begin{array}{cc}
a+c & b+d \\
-(b+d) & a+c
\end{array}\right) \in S, \\
k A=\left(\begin{array}{cc}
k a & k b \\
-k b & k a
\end{array}\right) \in S
\end{gathered}
$$

e

$$
A B=\left(\begin{array}{cc}
a c-b d & a d+b c \\
-b c-a d & -b d+a c
\end{array}\right)=\left(\begin{array}{cc}
a c-b d & a d+b c \\
-(a d+b c) & a c-b d
\end{array}\right) \in S
$$

Em linguagem técnica, isto significa que $S$ é fechado para a adição e multiplicação de matrizes e multiplicação por um escalar.

Agora, considere a função

$$
\begin{aligned}
\Psi: & \rightarrow \mathbb{C} \\
\left(\begin{array}{cc}
a & b \\
-b & a
\end{array}\right) & \mapsto a+b i .
\end{aligned}
$$

(i) $\Psi$ é uma função injetora, pois se $A=\left(\begin{array}{cc}a & b \\ -b & a\end{array}\right), B=\left(\begin{array}{cc}c & d \\ -d & c\end{array}\right) \in S$ são tais que $\Phi(A)=\Phi(B)$, então $a+b i=c+d i$ e pela igualdade de números complexos temos $a=c$ e $b=d$. Logo, $A=B$ e $\Phi$ é injetora.

(ii) $\Psi$ é uma função sobrejetora, pois dado $a+b i \in \mathbb{C}$, temos que $A=\left(\begin{array}{cc}a & b \\ -b & a\end{array}\right) \in S$ é tal que $\Psi(A)=a+b i$.

(iii) Para quaisquer $A, B \in S$ e $k \in \mathbb{R}, \Psi(A+B)=\Psi(A)+\Psi(B), \Psi(A B)=\Psi(A) \Psi(B)$ e $\Psi(k A)=k \Psi(A)$.

De fato, sejam $A=\left(\begin{array}{cc}a & b \\ -b & a\end{array}\right), C=\left(\begin{array}{cc}c & d \\ -d & c\end{array}\right) \in S$. Então:

$$
\Psi(A+C)=\Psi\left(\begin{array}{cc}
a+c & b+d \\
-b-d & a+c
\end{array}\right)=(a+c)+(b+d) i=(a+b i)+(c+d i)=\Psi(A)+\Psi(C) .
$$




$$
\begin{aligned}
\Psi(A C) & =\Psi\left(\begin{array}{cc}
a c+b(-d) & a d+b c \\
(-b) c+a(-d) & (-b) d+a c
\end{array}\right)=\Psi\left(\begin{array}{cc}
a c-b d & a d+b c \\
-(a d+b c) & a c-b d
\end{array}\right) \\
& =(a c-b d)+(a d+b c) i=a c-b d+a d i+b c i \\
& =a c+b d i^{2}+a d i+b c i=a c+b i d i+a d i+c b i \\
& =a(c+d i)+b i(c+d i)=(a+b i)(c+d i) \\
& =\Psi(A) \Psi(C) . \\
& \Psi(k A)=\Psi\left(\begin{array}{cc}
k a & k b \\
-k b & k a
\end{array}\right)=(k a)+(k b) i=k(a+b i)=k \Psi(A) .
\end{aligned}
$$

Os itens $(i)$ e (ii), mostram que a função $\Psi$ é uma bijeção entre $S$ e $\mathbb{C}$ e o item (iii) mostra que $\Psi$ preserva as adições e as multiplicações de $S$ e $\mathbb{C}$. O fato de $\Psi$ ser bijetora nos diz que há uma correspondência um a um entre os elementos de $S$ e de $\mathbb{C}$. Já o fato de $\Psi$ preservar as estruturas algébricas significa que quando operarmos em $\mathbb{C}$ estaremos operando automaticamente em $S$ e vice-versa. De maneira informal, isto significa que do ponto de vista algébrico, não há diferença em "fazer contas" em $\mathbb{C}$ ou em $S$.

$$
\begin{aligned}
& \text { Finalmente, se } A=\left(\begin{array}{cc}
a & b \\
-b & a
\end{array}\right) \in S \text {, então } \\
& \qquad\left(A^{t}\right)=\Psi\left(\begin{array}{cc}
a & -b \\
b & a
\end{array}\right)=a-b i=\overline{a+b i}=\overline{\Psi(A)} .
\end{aligned}
$$

Isto significa que "transpor" em $S$ é equivalente a "conjugar" em $\mathbb{C}$.

Encerramos esta seção observando que como $\Psi$ é bijetora, ela admite uma inversa $\Psi^{-1}: \mathbb{C} \rightarrow S$ que é dada por $\Psi^{-1}(a+b i)=\left(\begin{array}{cc}a & b \\ -b & a\end{array}\right)$, para qualquer $a+b i \in \mathbb{C}$.

\section{Norma e ângulos entre matrizes}

Motivados pelas funções $\Psi$ e $\Psi^{-1}$ da seção anterior e pelo fato de podermos definir módulo (ou norma) de um número complexo e ângulo entre números complexos, vamos mostrar como definir estes entes geométricos no conjunto das matrizes $\mathbb{M}_{2 \times 2}(\mathbb{R})$. Em seguida vamos mostrar que as funções $\Psi$ e $\Psi^{-1}$ preservam ângulos e módulo, ou seja, se $\theta$ é o ângulo entre duas matrizes $A, B \in S$, então $\theta$ será o ângulo entre os números complexos $\Psi(A)$ e $\Psi(B)$. Finalmente, se o módulo de uma matriz $A \in S$ é $d$, então o módulo de $\Psi(A)$ também é $d$. 
Definição 5.1. Sejam $A, B \in \mathbb{M}_{2 \times 2}(\mathbb{R})$ duas matrizes quaisquer. O número real $\frac{\operatorname{tr}\left(A^{t} B\right)}{2}$ (que é determinado unicamente pelas matrizes $A$ e $B$ ) é chamado um produto interno de $A$ por $B$ e denotado por $\langle A, B\rangle$.

$$
\begin{aligned}
& \text { Assim, se } A=\left(\begin{array}{ll}
a & b \\
c & d
\end{array}\right) \text { e } B=\left(\begin{array}{ll}
e & f \\
g & h
\end{array}\right) \text { são elementos de } \mathbb{M}_{2 \times 2}(\mathbb{R}) \text {, então } \\
& \langle A, B\rangle=\frac{1}{2} \operatorname{tr}\left(\left(\begin{array}{ll}
a & c \\
b & d
\end{array}\right)\left(\begin{array}{ll}
e & f \\
g & h
\end{array}\right)\right)=\frac{1}{2} \operatorname{tr}\left(\left(\begin{array}{ll}
a e+c g & a f+c h \\
b e+d g & b f+d h
\end{array}\right)\right)=\frac{a e+c g+b f+d h}{2} .
\end{aligned}
$$

Analogamente, $\langle B, A\rangle=\frac{a e+c g+b f+d h}{2}$. Portanto, $\langle A, B\rangle=\langle B, A\rangle$ é a soma dos produtos das respectivas entradas de $A$ e $B$. Em particular, $\left\langle A, A^{t}\right\rangle=\frac{a^{2}+2 b c+d^{2}}{2} \mathrm{e}\langle A, A\rangle=\frac{a^{2}+b^{2}+c^{2}+d^{2}}{2}=$ $\left\langle A^{t}, A^{t}\right\rangle$.

Exemplo 5.2. Para as matrizes $A=\left(\begin{array}{cc}-2 & 1 \\ -1 & -2\end{array}\right)$ e $B=\left(\begin{array}{cc}1 & -2 \\ 4 & 3\end{array}\right)$, temos

$$
\langle A, B\rangle=\frac{(-2) \cdot 1+1 \cdot(-2)+(-1) \cdot 4+(-2) \cdot 3}{2}=\frac{-14}{2}=-7 .
$$

Também

$$
\langle A, A\rangle=\frac{(-2)^{2}+1^{2}+(-1)^{2}+(-2)^{2}}{2}=5 \text { e }\langle B, B\rangle=\frac{1^{2}+(-2)^{2}+4^{2}+3^{2}}{2}=\frac{30}{2}=15 .
$$

Exemplo 5.3. Se $A=\left(\begin{array}{ll}2 & 1 \\ 1 & 1\end{array}\right)$ e $B=\left(\begin{array}{cc}-1 & 2 \\ 2 & -2\end{array}\right)$, então

$$
\langle A, B\rangle=\frac{2 \cdot(-1)+1 \cdot 2+1 \cdot 2+1 \cdot(-2)}{2}=\frac{0}{2}=0 .
$$

Proposição 5.4. As seguintes propriedades são válidas para quaisquer matrizes $A, B, C \in \mathbb{M}_{2 \times 2}(\mathbb{R}) \boldsymbol{e} \alpha \in \mathbb{R}$.

(i) $\langle A, A\rangle \geq 0$ e $\langle A, A\rangle=0$ se, e somente se, $A=0$;

(ii) $\langle\alpha A, B\rangle=\alpha\langle A, B\rangle$;

(iii) $\langle A+B, C\rangle=\langle A, C\rangle+\langle B, C\rangle$;

iv) $\langle A, B\rangle=\langle B, A\rangle$. 
Demonstração. Sejam $A=\left(\begin{array}{ll}a & b \\ c & d\end{array}\right), B=\left(\begin{array}{ll}e & f \\ g & h\end{array}\right)$ e $C=\left(\begin{array}{ll}x & y \\ z & w\end{array}\right) \in \mathbb{M}_{2 \times 2}(\mathbb{R})$ e $\alpha \in \mathbb{R}$.

(i) Conforme vimos anteriormente, $\langle A, A\rangle=\frac{a^{2}+b^{2}+c^{2}+d^{2}}{2}$ e como $a^{2}, b^{2}, c^{2}, d^{2} \geq 0$, segue que $\langle A, A\rangle \geq 0$. Mais ainda: $\langle A, A\rangle=0$ se, e somente se, $a=b=c=d=0$.

(ii) Temos

$$
\begin{aligned}
\langle\alpha A, B\rangle & =\frac{1}{2} \operatorname{tr}\left(\left(\begin{array}{ll}
\alpha a & \alpha b \\
\alpha c & \alpha d
\end{array}\right)^{t}\left(\begin{array}{ll}
e & f \\
g & h
\end{array}\right)\right)=\frac{1}{2} \operatorname{tr}\left(\left(\begin{array}{ll}
\alpha a & \alpha c \\
\alpha b & \alpha d
\end{array}\right)\left(\begin{array}{ll}
e & f \\
g & h
\end{array}\right)\right) \\
& =\frac{1}{2} \operatorname{tr}\left(\alpha\left(\begin{array}{ll}
a & b \\
c & d
\end{array}\right)^{t}\left(\begin{array}{ll}
e & f \\
g & h
\end{array}\right)\right)=\alpha \frac{1}{2} \operatorname{tr}\left(\left(\begin{array}{ll}
a & b \\
c & d
\end{array}\right)^{t}\left(\begin{array}{ll}
e & f \\
g & h
\end{array}\right)\right) \\
& =\alpha\langle A, B\rangle
\end{aligned}
$$

em que a penúltima igualdade segue da Proposição 3.11 (iii)

(iii) Por um lado,

$$
\begin{aligned}
& \langle A+B, C\rangle=\left\langle\left(\begin{array}{cc}
a+e & b+f \\
c+g & d+h
\end{array}\right),\left(\begin{array}{cc}
x & y \\
z & w
\end{array}\right)\right\rangle=\frac{1}{2} \operatorname{tr}\left(\left(\begin{array}{cc}
a+e & b+f \\
c+g & d+h
\end{array}\right)^{t}\left(\begin{array}{ll}
x & y \\
z & w
\end{array}\right)\right) \\
& =\frac{1}{2} \operatorname{tr}\left(\left(\begin{array}{cc}
a+e & c+g \\
b+f & d+h
\end{array}\right)\left(\begin{array}{ll}
x & y \\
z & w
\end{array}\right)\right) \\
& =\frac{1}{2} \operatorname{tr}\left(\left(\begin{array}{cc}
(a+e) x+(c+g) z & (a+e) y+(c+g) w \\
(b+f) x+(d+h) z & (b+f) y+(d+h) w
\end{array}\right)\right) \\
& =\frac{1}{2} \operatorname{tr}\left(\left(\begin{array}{cc}
a x+e x+c z+g z & a y+e y+c w+g w \\
b x+f x+d z+h z & b y+f y+d w+h w
\end{array}\right)\right) \\
& =\frac{(a x+e x+c z+g z)+(b y+f y+d w+h w)}{2} \\
& =\frac{(a x+c z)+(e x+g z)+(b y+d w)+(f y+h w)}{2} .
\end{aligned}
$$


Por outro lado,

$$
\begin{aligned}
\langle A, C\rangle+\langle B, C\rangle & =\frac{1}{2} \operatorname{tr}\left(\left(\begin{array}{ll}
a & b \\
c & d
\end{array}\right)^{t}\left(\begin{array}{ll}
x & y \\
z & w
\end{array}\right)\right)+\frac{1}{2} \operatorname{tr}\left(\left(\begin{array}{ll}
e & f \\
g & h
\end{array}\right)^{t}\left(\begin{array}{ll}
x & y \\
z & w
\end{array}\right)\right) \\
& =\frac{1}{2} \operatorname{tr}\left(\left(\begin{array}{ll}
a & c \\
b & d
\end{array}\right)^{\prime}\left(\begin{array}{ll}
x & y \\
z & w
\end{array}\right)\right)+\frac{1}{2} \operatorname{tr}\left(\left(\begin{array}{ll}
e & g \\
f & h
\end{array}\right)\left(\begin{array}{ll}
x & y \\
z & w
\end{array}\right)\right) \\
& =\frac{1}{2} \operatorname{tr}\left(\left(\begin{array}{ll}
a x+c z & a y+c w \\
b x+d z & b y+d w
\end{array}\right)\right)+\frac{1}{2} \operatorname{tr}\left(\left(\begin{array}{ll}
e x+g z & e y+g w \\
f x+h z & f y+h w
\end{array}\right)\right) \\
& =\frac{(a x+c z)+(e x+g z)}{2}+\frac{(b y+d w)+(f y+h w)}{2} .
\end{aligned}
$$

Logo,

$$
\langle A+B, C\rangle=\frac{(a x+c z)+(e x+g z)+(b y+d w)+(f y+h w)}{2}=\langle A, C\rangle+\langle B, C\rangle
$$

e temos o desejado.

(iv) Já foi mostrado anteriormente.

Como acabamos de ver no item $(i)$, para toda matriz $A \in \mathbb{M}_{2 \times 2}(\mathbb{R}),\langle A, A\rangle$ é um número real maior ou igual a zero. Por isso, podemos calcular $\sqrt{\langle A, A\rangle}$. Este número é definido como sendo o módulo ou norma da matriz $A$ e é denotado por $\|A\|$. Observe que $\left\|A^{t}\right\|=\sqrt{\left\langle A^{t}, A^{t}\right\rangle}=\sqrt{\langle A, A\rangle}=$ $\|A\|$.

$$
\begin{aligned}
& \text { Desta forma, se } A=\left(\begin{array}{ll}
a & b \\
c & d
\end{array}\right) \in \mathbb{M}_{2 \times 2}(\mathbb{R}) \text {, então } \\
& \|A\|=\sqrt{\langle A, A\rangle}=\sqrt{\frac{a^{2}+b^{2}+c^{2}+d^{2}}{2}}=\frac{\sqrt{a^{2}+b^{2}+c^{2}+d^{2}}}{\sqrt{2}}=\frac{\sqrt{2}}{2} \sqrt{a^{2}+b^{2}+c^{2}+d^{2}} . \\
& \text { Em particular, para qualquer } A=\left(\begin{array}{cc}
a & b \\
-b & a
\end{array}\right) \in S, \text { temos } \\
& \qquad\langle A, A\rangle=\frac{\left(a^{2}+b^{2}\right)+\left((-b)^{2}+a^{2}\right)}{2}=\frac{2 a^{2}+2 b^{2}}{2}=a^{2}+b^{2} .
\end{aligned}
$$

e, com isso,

$$
\|A\|=\sqrt{a^{2}+b^{2}}
$$


Disso e de $\Psi(A)=a+b i$, temos

$$
\|A\|=|\Psi(A)|
$$

Portanto, mostramos que além da estrutura algébrica, a função $\Psi$ preserva a norma.

Exemplo 5.5. Considere $A=\left(\begin{array}{cc}2 & 3 \\ -3 & 2\end{array}\right), B=\left(\begin{array}{cc}3 & -4 \\ 4 & 3\end{array}\right) \in \operatorname{S.Então} \Psi(A)=2+3 i, \Psi(B)=3-4 i$ $e\|A\|=|2+3 i|=\sqrt{2^{2}+3^{2}}=\sqrt{13}$ e $\|B\|=|3-4 i|=\sqrt{25}=5$.

O próximo resultado traz algumas propriedades do produto interno definido anteriormente. Isso nos permitirá definir ângulo entre matrizes.

Proposição 5.6. Para quaisquer $A, B \in \mathbb{M}_{2 \times 2}(\mathbb{R})$ e $\alpha \in \mathbb{R}$, são válidas:

i) $\|A\| \geq 0$ e $\|A\|=0$ se, e somente se $A=0$.

ii) $\|\alpha A\|=|\alpha|\|A\|$.

iii) (Desigualdade de Schwarz) $|\langle A, B\rangle| \leq\|A\| \cdot\|B\|$.

iv) (Desigualdade Triangular) $\|A+B\| \leq\|A\|+\|B\|$.

Demonstração. i) Segue diretamente da Proposição $5.4(i)$ e do fato de $\|A\|=\sqrt{\langle A, A\rangle}$.

ii) Sejam $\alpha \in \mathbb{R}$ e $A \in \mathbb{M}_{2 \times 2}$ (R). Então, pela Proposição 5.4 (ii) e pela definição de norma,

$$
\begin{aligned}
\|\alpha A\| & =\sqrt{\langle\alpha A, \alpha A\rangle}=\sqrt{\alpha\langle A, \alpha A\rangle} \\
& =\sqrt{\alpha\langle\alpha A, A\rangle}=\sqrt{\alpha \alpha\langle A, A\rangle} \\
& =\sqrt{\alpha^{2}\langle A, A\rangle}=\sqrt{\alpha^{2}} \sqrt{\langle A, A\rangle} \\
& =|\alpha|\|A\| .
\end{aligned}
$$

iii) Sejam $A, B \in \mathbb{M}_{2 \times 2}(\mathbb{R})$.

Se $A=0$ ou $B=0$, valem as igualdades $|\langle A, B\rangle|=0=\|A\| \cdot\|B\|$.

Suponhamos que $A \neq 0$ e $B \neq 0$. Para qualquer $\alpha \in \mathbb{R}$, temos da Proposição 5.4 (iii) e (iv) e dos itens $(i)$ e $(i i)$ anteriores que:

$$
\begin{aligned}
0 & \leq\langle\alpha A+B, \alpha A+B\rangle=\langle\alpha A, \alpha A+B\rangle+\langle B, \alpha A+B\rangle \\
& =\langle\alpha A+B, \alpha A\rangle+\langle\alpha A+B, B\rangle=\langle\alpha A, \alpha A\rangle+\langle B, \alpha A\rangle+\langle\alpha A, B\rangle+\langle B, B\rangle \\
& =\alpha^{2}\langle A, A\rangle+2 \alpha\langle A, B\rangle+\langle B, B\rangle=\langle A, A\rangle \alpha^{2}+2\langle A, B\rangle \alpha+\langle B, B\rangle
\end{aligned}
$$


Como o número real $\langle A, A\rangle$ é positivo (pois $A \neq 0$ ), obtemos uma inequação de grau 2 em $\alpha$. Neste caso, $\Delta \leq 0$, ou seja,

$$
4\langle A, B\rangle^{2}-4\langle A, A\rangle\langle B, B\rangle=4\langle A, B\rangle^{2}-4\|A\|^{2} \cdot\|B\|^{2} \leq 0 .
$$

Isto implica que

$$
\langle A, B\rangle^{2} \leq\|A\|^{2} \cdot\|B\|^{2}
$$

ou ainda,

$$
|\langle A, B\rangle|=\sqrt{\langle A, B\rangle^{2}} \leq \sqrt{\|A\|^{2} \cdot\|B\|^{2}}=\|A\| \cdot\|B\| .
$$

Vale lembrar que $\sqrt{\langle A, B\rangle^{2}}$ é igual ao módulo de $\langle A, B\rangle$ e não necessariamente igual $\langle A, B\rangle$, pois $\langle A, B\rangle$ pode ser um número negativo, conforme Exemplo 5.2 .

iv) Sejam $A, B \in \mathbb{M}_{2 \times 2}(\mathbb{R})$. Pela Proposição 5.4(iii) e (iv), pelo item (iii) anterior e pela definição de norma, temos:

$$
\begin{aligned}
\|A+B\|^{2} & =(\sqrt{\langle A+B, A+B\rangle})^{2}=\langle A+B, A+B\rangle \\
& =\|A\|^{2}+2\langle A, B\rangle+\|B\|^{2} \leq\|A\|^{2}+2|\langle A, B\rangle|+\|B\|^{2} \\
& \leq\|A\|^{2}+2\|A\|\|B\|+\|B\|^{2} \\
& =(\|A\|+\|B\|)^{2} .
\end{aligned}
$$

Como $\|A+B\|$ e $\|A\|+\|B\|$ são números $\geq 0$, podemos extrair a raiz quadrada de ambos os lados sem alterar a desigualdade. Logo, $\|A+B\| \leq\|A\|+\|B\|$.

Sejam $A, B \in \mathbb{M}_{2 \times 2}(\mathbb{R})$ duas matrizes não nulas. Pela Desigualdade de Schwarz, $|\langle A, B\rangle| \leq$ $\|A\| \cdot\|B\|$. Portanto, por uma propriedade de módulo, $-(\|A\| \cdot\|B\|) \leq\langle A, B\rangle \leq\|A\| \cdot\|B\|$, isto é,

$$
-1 \leq \frac{\langle A, B\rangle}{\|A\| \cdot\|B\|} \leq 1
$$

Como a função $\cos :[0, \pi] \longrightarrow[-1,1]$ é bijetora, existe um único $\theta \in[0, \pi]$ tal que

$$
\cos (\theta)=\frac{\langle A, B\rangle}{\|A\| \cdot\|B\|} .
$$

Tal $\theta$ é definido como sendo ângulo entre as matrizes $A$ e $B$ e será denotado por $\theta_{A, B}$.

$$
\begin{aligned}
\text { Em particular, se } A=\left(\begin{array}{cc}
a & b \\
-b & a
\end{array}\right) \text { e } B=\left(\begin{array}{cc}
c & d \\
-d & c
\end{array}\right) \text {, então } \\
\qquad \cos \left(\theta_{A, B}\right)=\frac{\langle A, B\rangle}{\|A\| \cdot\|B\|}=\frac{(a c+b d)}{\sqrt{a^{2}+b^{2}} \cdot \sqrt{c^{2}+d^{2}}} .
\end{aligned}
$$


Por outro lado, se $z_{1}=\Psi(A)=a+b i$ e $z_{2}=\Psi(B)=c+d i$, então

$$
\cos \left(\theta_{z_{1}, z_{2}}\right)=\frac{a c+b d}{\sqrt{a^{2}+b^{2}} \cdot \sqrt{c^{2}+d^{2}}}=\cos \left(\theta_{A, B}\right) .
$$

Logo, o ângulo entre $A, B \in \mathbb{M}_{2 \times 2}(\mathbb{R})$ e $\Psi(A), \Psi(B) \in \mathbb{C}$ coincidem.

Exemplo 5.7. Consideremos as matrizes $A=\left(\begin{array}{cc}2 & 4 \\ 3 & -1\end{array}\right)$ e $B=\left(\begin{array}{ll}3 & 5 \\ 2 & 1\end{array}\right)$. Então,

$$
\langle A, B\rangle=\frac{31}{2},\langle A, A\rangle=\frac{30}{2}=15 \text { e }\langle B, B\rangle=\frac{39}{2} .
$$

Daí,

$$
\cos \left(\theta_{A, B}\right)=\frac{31 / 2}{\sqrt{15} \cdot \sqrt{39 / 2}} \approx 0,9062
$$

Portanto, $\theta_{A, B} \approx 43,61^{\circ}$.

Exemplo 5.8. Para as matrizes $A$ e $B$ do Exemplo 5.3, temos $\langle A, B\rangle=0$ e, portanto, $\cos \left(\theta_{A, B}\right)=0$, ou seja, $\theta_{A, B}=90^{\circ}$.

Exemplo 5.9. Seja $A=\left(\begin{array}{ll}a & b \\ c & d\end{array}\right)$. Então, $A^{t}=\left(\begin{array}{ll}a & c \\ b & d\end{array}\right) e,\left\langle A, A^{t}\right\rangle=\frac{a^{2}+2 b c+d^{2}}{2} \boldsymbol{e}\|A\|=$ $\sqrt{\frac{a^{2}+b^{2}+c^{2}+d^{2}}{2}}=\left\|A^{t}\right\|$. Logo, $\cos \left(\theta_{A, A^{t}}\right)=\frac{a^{2}+2 b c+d^{2}}{\left(a^{2}+b^{2}+c^{2}+d^{2}\right)}$. Em particular, se $c=a$ e $d=b$, então $\left\langle A, A^{t}\right\rangle=\frac{(a+b)^{2}}{2}$ e $\cos \left(\theta_{A, A^{t}}\right)=\frac{(a+b)^{2}}{2\left(a^{2}+b^{2}\right)}$. Também se $d=a \boldsymbol{e} c=-b$, ou seja, se $A \in S$, então $\left\langle A, A^{t}\right\rangle=a^{2}-b^{2}$ e $\cos \left(\theta_{A, A^{t}}\right)=\frac{a^{2}-b^{2}}{a^{2}+b^{2}}$.

Observação 5.10. Encerramos a seção observando que na Definição 5.1 poderíamos ter definido o produto interno de $A, B \in \mathbb{M}_{2 \times 2}(\mathbb{R})$ como sendo $\langle A, B\rangle_{k}=k \operatorname{tr}\left(A^{t} B\right)$, em que $k$ é um número real positivo qualquer. É fácil ver que todas as propriedades da Proposição 5.4 continuam válidas, pois na demonstração o número $\frac{1}{2}$ pode ser substituído por $k$ sem qualquer prejuízo. Neste caso, podemos definir a norma de $A$ como sendo $\|A\|_{k}=\sqrt{\langle A, A\rangle_{k}}$ e podemos mostrar que todas as propriedades da Proposição 5.6 também são verdadeiras. Mais ainda, cálculos simples mostram que $\langle A, B\rangle_{k}=(2 k) \frac{1}{2} \operatorname{tr}\left(A^{t} B\right)=2 k\langle A, B\rangle \boldsymbol{e}\|A\|_{k}=\sqrt{2 k}\|A\|$. Assim, fica claro que cada valor $k$ nos dá uma norma diferente. Porém, o ângulo entre duas matrizes não muda para diferentes valores de $k$, pois na Equação 3 , temos

$$
\frac{\langle A, B\rangle_{k}}{\|A\|_{k} \cdot\|B\|_{k}}=\frac{2 k\langle A, B\rangle}{\sqrt{2 k}\|A\| \cdot \sqrt{2 k}\|B\|}=\frac{\langle A, B\rangle}{\|A\| \cdot\|B\|}=\cos (\theta)
$$


Finalmente, observe que se $A \in S$ então $\|A\|_{k}=\sqrt{2 k}\|A\|=\sqrt{2 k}\|\Psi(A)\|$. Com isso, concluímos que o fato de multiplicarmos $\operatorname{tr}\left(A^{t} B\right)$ por $\frac{1}{2}$ é primordial para que a função $\Psi$ preserve a norma.

\section{Considerações finais}

As noções geométricas de norma e ângulo definidas em $\mathbb{C}$ e a correspondência bijetora $\Psi$ entre $\mathbb{C}$ e o subconjunto $S$ das matrizes $2 \times 2$ foram nossa inspiração para tentar definir os mesmos entes geométricos no conjunto das matrizes $M_{2 \times 2}(\mathbb{R})$. Isto é extremamente interessante, pois mostra que temos uma geometria num conjunto cujos elementos não têm representação geométrica. De fato, ver uma matriz, por exemplo, como uma flecha orientada pode ser uma tarefa um tanto quanto difícil. A mesma dificuldade pode ser encontrada quando tentamos visualizar o ângulo entre duas matrizes. Podemos concluir desta forma que a geometria não depende de boas representações (desenhos), mas sim de definições precisas e raciocínio lógico adequado.

Ressaltamos também o fato da função $\Psi$ preservar ângulo e norma (além de adição e multiplicação), mostrando que as definições de ângulo e norma em $M_{2 \times 2}(\mathbb{R})$ são elegantes e precisas. Também observamos que na definição de produto interno de matrizes, multiplicamos o traço por $\frac{1}{2}$. Durante a pesquisa percebemos que este "ajuste" era necessário para que a função $\Psi$ preservasse ângulos e norma. Sugerimos Coelho e Lourenço (2007) para quem estiver interessado em estudar produto interno e norma para casos mais gerais.

Não podemos deixar de mencionar a importância das propriedades apresentadas na Proposição 5.6. que foram o alicerce para a definição de ângulo. $O$ fato da função $\cos :[0, \pi] \longrightarrow[-1,1]$ ser bijetora foi a chave mestra para definirmos a unicidade do ângulo entre matrizes.

Embora os assuntos abordados nas duas últimas seções tenham um grau maior de complexidade, nós tentamos, na medida do possível, escrever em uma linguagem mais simples a fim de que mais pessoas possam ler sem a exigência de dominar assuntos avançados de matemática. Nosso objetivo é instigar o leitor a estudar uma pequena parte da matemática que vai além dos conteúdos do ensino regular. Para tanto, evitamos ao máximo expressões de matemática avançada, tais como Espaços Vetoriais, Transformações Lineares, Isomorfismos etc. Porém, esses e outros conceitos estão nas entrelinhas do texto. 
Finalmente, os cálculos que apresentamos, muitas vezes são mais tediosos que difíceis. Por isso, acreditamos que este conteúdo pode ser trabalhado com alunos de Ensino Médio que tenham interesse em explorar um pouco sobre matemática não contida nos livros da Educação Básica.

\section{Referências}

BOLDRINI, J. L.; COSTA, S. I. R.; FIGUEIREDO, V. L.; WETZLER, H. G. Álgebra Linear I. 3. ed. São Paulo: Harper \& Row do Brasil, 1986.

CAETANO, D. M. As Diferentes Faces dos Números Complexos. 2018. 87 f. Dissertação (Mestrado em Matemática) - Programa de Mestrado Profissional em Matemática em Rede Nacional, Universidade Estadual de Maringá, Maringá, 2018.

CALLIOLI, C. S.; DOMINGUES, H. H.; COSTA, R. C. F. Álgebra Linear e Aplicações. 2. ed. São Paulo: Atual, 1978.

COELHO, F. U.; LOURENÇO, M. L. Um Curso de Álgebra Linear. 2. ed. São Paulo: Editora da Universidade de São Paulo, 2007.

FERNANDES, C. S.; BERNARDES, N. C. J. Introdução às Funções de uma Variável Complexa. 2. ed. Rio de Janeiro: SBM, 2008.

GARBI, Gilberto G. O romance das equações algébricas. 3. ed. rev. ampl. São Paulo: Livraria da Física. 2009.

GILBERTO, G. G. O Romance das Equações Algébricas. 3. ed. São Paulo: Editora Livraria da Física, 2009.

SILVA, S. M. da. Números complexos: uma abordagem matricial. 2017. 74 f. Dissertação (Mestrado em Matemática) - Programa de Mestrado Profissional em Matemática em Rede Nacional, Universidade Federal de Roraima, Boa Vista, 2017.

SOARES, M. G. Cálculo em uma variável complexa. 5. ed. Rio de Janeiro: IMPA, 2009. 